\title{
Heartbeat: Altmetrics and Cardiovascular Risk Factor Studies
}

The main guiding editorial principle of Heart is to improve the care of patients with cardiovascular disease by publishing high-impact innovative clinically relevant original cardiovascular research. However, it can be difficult to specify exactly what defines "high-impact". In addition to the traditional Impact Factor (Journal Citation Reports ${ }^{\circledR}$, Thomson Reuters, 2015) used for medical journals, there are many other proposed measures of influence, including the Almetric (Altmetric. com) score. The Altmetric score, shown as a number within a colored wreath at the end of each full text article online, reflects the immediate attention received by a research paper as evidenced by articles in news outlets and commentary on blogs, as well as the number of tweets and other digital communications (figure 1).

As you know, the traditional Impact Factor (Thompson-Reuters) averages the annual number of citations of papers published in a journal over the previous 2 years, normalized to the total number of publications. Thus, the Impact Factor reflects past publications, groups all the papers in a journal together and only considers citations by other scientific journals. In contrast, the Almetrics score provides immediate feedback about an individual article and indicates the public (as well as scientific) interest in the research findings. In this issue of Heart, an article on sweetened beverage consumption and the risk of heart failure in men (see page 1961) has one of the highest ever Altmetrics scores for Heart papers and is in the top $5 \%$ of all research outputs scored by Altmetrics. Interestingly, $89 \%$ of the score is based on interest by the public, $7 \%$ by clinicians, and $4 \%$ by scientists. Authors and readers can see the geographical and demographic breakdown of the score for any paper by clicking on details on the webpage. Perhaps, as authors and readers, we should pay more attention to these scores. We all agree that the goal of medical research is to improve patient outcomes. Now, we should start listening to our patients' concerns as reflected by public interest in journal articles.
Correspondence to Professor Catherine M Otto, Division of Cardiology, University of Washington, Seattle, WA 98195, USA; cmotto@u.washington.edu

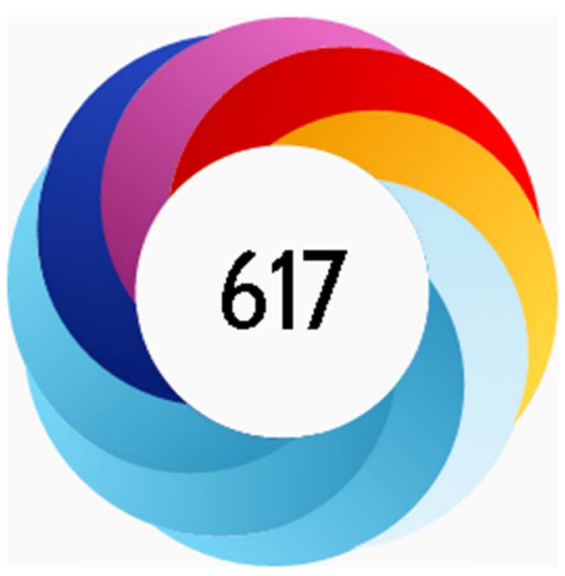

Figure 1 Example of an Altmetric score. The colors in the wreath represent the relative contributions of different types of interest (news outlets, twitter, blogs, etc) with the number in the middle indicating the interest in this article, relative to other articles. (from Heart webpage for this article)

In a population based cohort of over 40 thousand men between 45 and 79 years of age, followed for a mean of 11.7 years, consumption of two or more sweetened beverages per day was associated with a 23\% higher risk of developing heart failure (HF), after adjustment for standard cardiovascular risk factors and other dietary factors (see page 1961). In this study, sweetened beverages included both sugar sweetened and artificially sweetened drinks, but not fruit juice. In the accompanying editorial, MartinezGonzález and Ruiz-Canela (see page 1935) (figure 2) argue that more research on nutritional factors associated with HF is needed given the prevalence and mortality of HF and the increased risk of HF in patients who are obese or have type- 2 diabetes. A detailed table summarizes the risk of $\mathrm{HF}$ relative to the elements included in a healthy life-style score. Taken together, these data suggest that sweetened beverages "are usually components of a poor quality dietary pattern and that overall dietary patterns better represent the broader picture of food habits and are more important determinants of disease than any isolated food or beverage." The authors conclude: "the advice to the general population should be that their most sensible option will be to reduce or eliminate their consumption of sweetened beverages, replacing them with water to comply with the requirements for good hydration."

Environmental factors associated with cardiovascular disease (CVD) mortality include the weather. In a very large study of the effects of temperature on CVD mortality in cities in China, approximately $17 \%$ of CVD mortality was attributable to ambient temperature with the magnitude of this effect varying between cities (see page 1966) (figure 3). Cold weather accounted from most of the increased risk (15.8\%), with hot weather accounting for a much smaller effect (1.3\%).

Marti-Soler and Marques-Vidal (see page 1941) discuss potential mechanisms for the association between ambient temperature and CVD mortality. Possible explanations include seasonable variation in CVD risk factors, seasonal viral infections which might trigger CVD, life-style

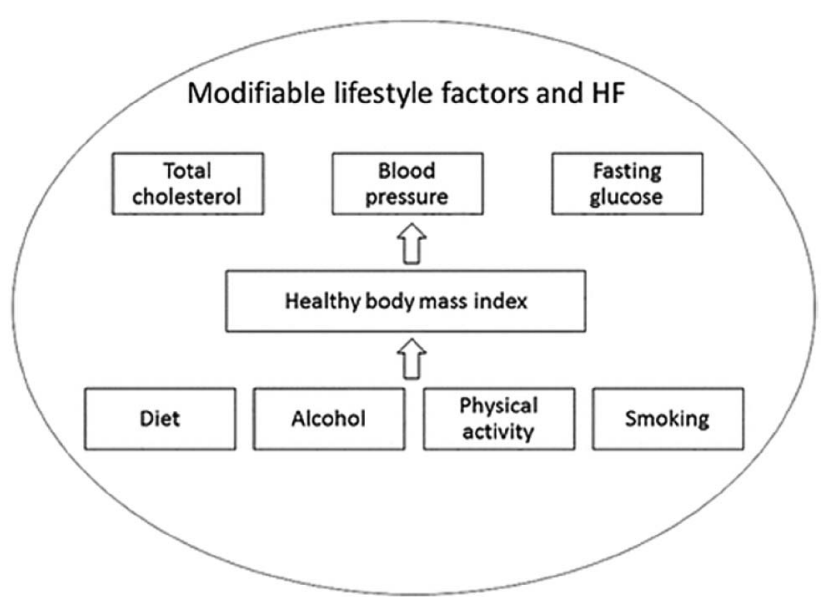

Figure 2 Relationship between lifestyle factors associated with heart failure. 


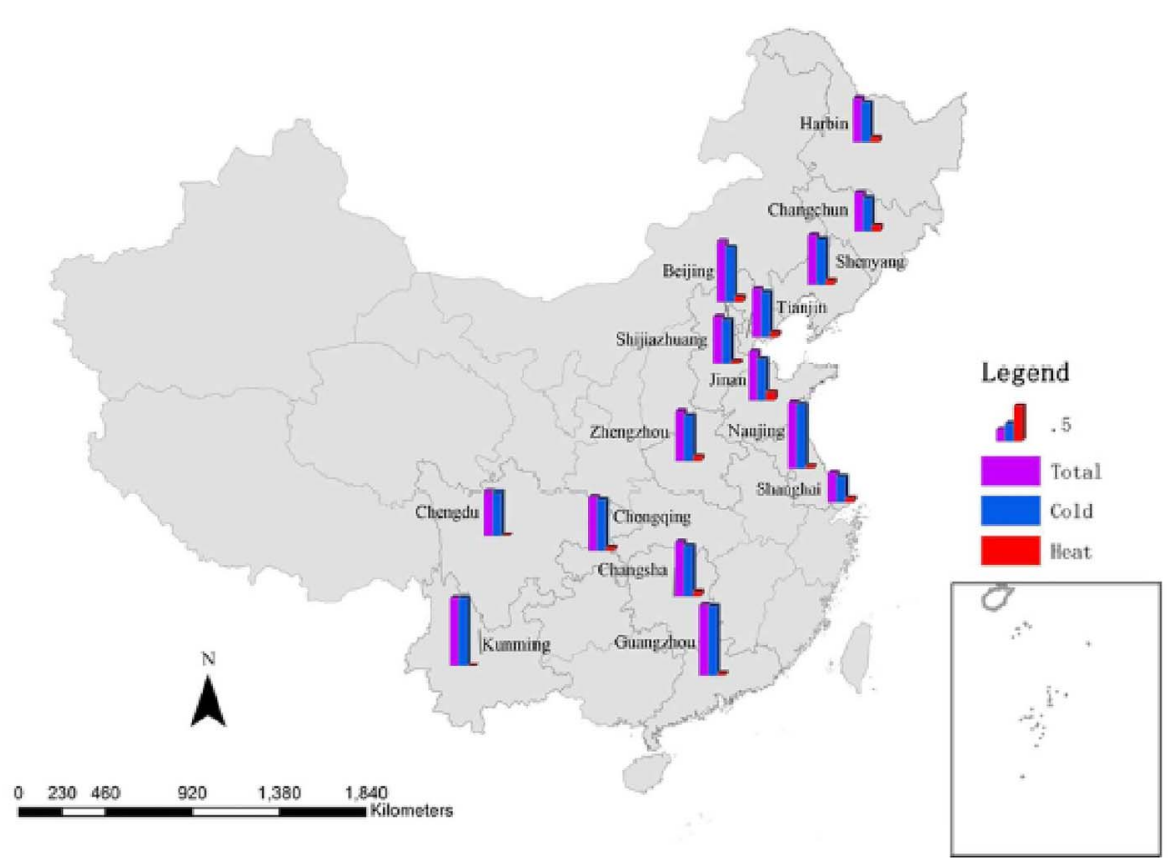

Figure 3 The locations of 15 Chinese cities in this study, with attributable cardiovascular mortality fraction computed as total and as separated components for cold and hot temperatures.

changes during cold weather including decreased physical activity, and other environmental factors such as increased outdoor or indoor air pollution. On the other hand, it seems counterintuitive that the effects of cold temperature were greater in the warmer southern cities than the colder northern cities, suggesting that the effects of temperature were modulated

by living conditions or cold-coping behaviors. A better understanding of the specific factors underlying the association between cold weather and heart disease is needed to guide public policy toward effective and affordable strategies to reduce CVD mortality.

Many children born with congenital heart disease now survive to adulthood,

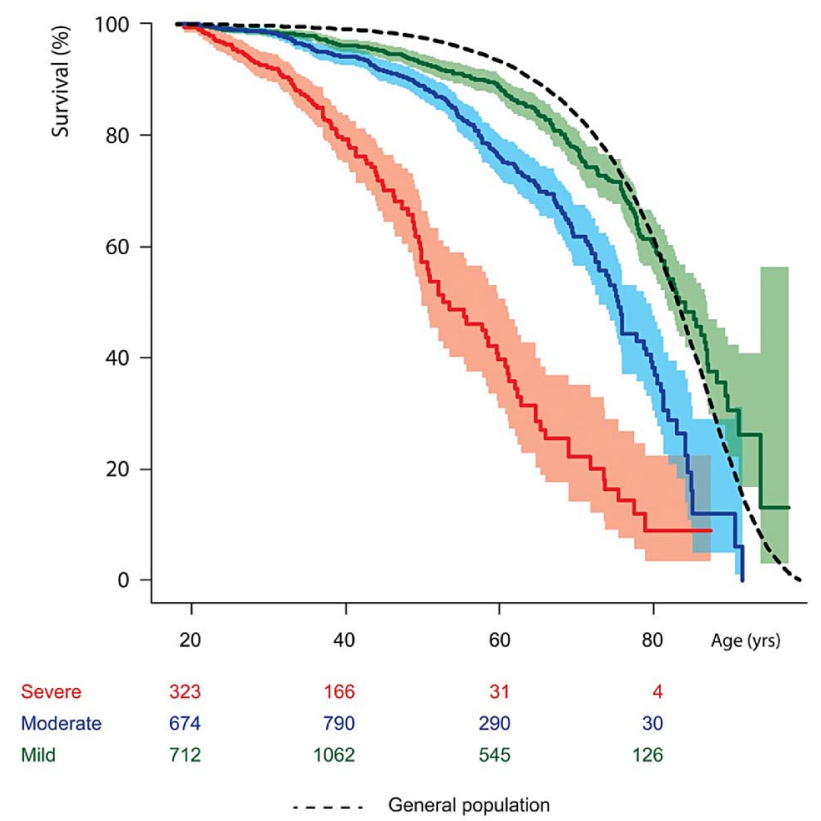

typically after palliative or curative invention. However, accurate data on the longterm survival of these patients has been lacking which hampers appropriate allocation of resources and hinders ensuring that enough health care providers receive education and training in adult congenital heart disease (ACHD). Using the national registry of ACHD patients in the Netherlands, Bouma and colleagues (see page 1989) found a median survival of about 53 years for patients with severe heart lesions and 75 years for those with moderate lesions. ACHD patients with mild residual disease had a median survival about 84 years, similar to the general population (figure 4). This data further supports the need for increasing the health care workforce with expertise in ACHD, along with additional centers of excellence in management of ACHD. Modeling of the expected numbers of patients at the country and regional levels are needed to appropriately meet this need.

The Education in Heart article (see page 1996) in this issue is entitled "When to consider an implantable cardiac defibrillator (ICD) following myocardial infarction?" This concise review summarizes the evidence and current guideline recommendations. The authors' answer to the title question is: "Primary prevention ICDs should be considered for patients who have severe LV systolic dysfunction despite 3 months of optimal medical therapy in ischaemic or non-ischaemic cardiomyopathy (as per ESC and NICE guidelines). Patients should also have an anticipated life expectancy of $>1$ year with a good quality of life before consideration is made for ICD therapy. Furthermore, it is important to target patients deemed to be at highest risk of sudden death."

The Image Challenge case (see page 1953) shows a "ring in the heart" on echocardiographic imaging with pathological confirmation of this interesting and unusual diagnosis. See if you can identify the abnormality yourself.

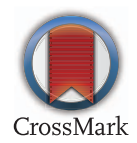

To cite Otto CM. Heart 2015;101:1931-1932.

Heart 2015;101:1931-1932.

doi:10.1136/heartjnl-2015-308995

Figure 4 Kaplan-Meier analysis for adult congenital heart disease patients stratified by severity of residual disease; dashed line represents survival of the general population, and shaded areas represent the $95 \% \mathrm{Cl}$ of the survival curves. 